\title{
Efeito da estimulação elétrica nervosa transcutânea no tratamento da dor no ombro de pacientes com doença renal crônica: relato de casos

\author{
Effect of transcutaneous electrical nerve stimulation in the treatment of pain in the \\ shoulder of patients with chronic kidney disease: a two-case report
}

\author{
Patrícia Ribeiro Bertoli', Vanda Jorgetti², Rosa Maria Affonso Moysés', \\ Rubens Correa Araújo, Celso Ricardo Fernandes Carvalho ${ }^{4}$
}

\author{
Estudo desenvolvido no \\ Ambulatório de Osteodistrofia \\ Renal do Hospital das Clínicas \\ da FMUSP - Faculdade de \\ Medicina da Universidade de \\ São Paulo, São Paulo, SP, Brasil \\ 1 Fisioterapeuta Ms. \\ 2 Médica da Divisão de \\ Nefrologia da FMUSP \\ 3 Prof. Dr. do Depto. de \\ Fisioterapia da Universidade \\ São Judas, São Paulo, SP \\ 4 Prof. Dr. do Depto. de \\ Fisioterapia, Fonoaudiologia e \\ Terapia Ocupacional da \\ FMUSP

\section{ENDEREÇO PARA} \\ CORRESPONDÊNCIA \\ Celso R. F. Carvalho \\ Faculdade de Medicina, \\ Universidade de São Paulo \\ Av. Dr. Arnaldo 455 sala 1216 \\ 01246-903 São Paulo SP \\ e-mail: cscarval@usp.br; \\ patybertoli@ig.com.br \\ APRESENTAÇÃo \\ dez. 2008 \\ ACEITO PARA PUBLICAÇÃO \\ jun. 2009
}

Resumo: A doença renal crônica (DRC) pode causar muitas complicações clínicas associadas ao tempo de diálise. A amiloidose é uma dessas complicações e pode levar à dor no ombro, comum em pacientes com DRC. Só foram encontrados na literatura relatos de tratamento cirúrgico e/ou medicamentoso para esse tipo de dor. O objetivo aqui foi avaliar o benefício da estimulação elétrica nervosa transcutânea (TENS) no tratamento da dor no ombro devida à amiloidose em pacientes com DRC. Duas pacientes foram avaliadas quatro vezes num período de três meses. Na avaliação inicial as pacientes foram orientadas e receberam um aparelho de TENS para uso domiciliar $(10 \mathrm{~Hz}, 150 \mu \mathrm{s}$, duas vezes por dia, por 40 minutos) durante três meses. A dor foi avaliada utilizando-se uma escala visual analógica e o questionário de dor de Wisconsin. Os resultados mostram que o tratamento com TENS reduziu a dor no ombro em ambas as pacientes. O tratamento com a TENS pode pois ser eficaz em reduzir a dor no ombro em pacientes com DRC.

DEscritoREs: Amiloidose; Dor de ombro; Insuficiência renal crônica; Terapia por estimulação elétrica

ABSTRACT: Chronic kidney disease (CKD) may bring about several clinical complications associated to long-term dialysis. Amyloidosis is one of such complications and may lead to shoulder pain, common among CKD patients, for which no studies could be found in literature on the use of transcutaneous electrical nerve stimulation (TENS) - only surgical and medicine treatments are reported. The purpose here was to assess benefits of TENS in treating shoulder pain due to amyloidosis in patients with CKD. Two female patients were assessed four times in a three-month period. At the initial evaluation, patients were guided as to its use and given a TENS device for use at home $(10 \mathrm{~Hz}, 150 \mu \mathrm{s}$, twice a day, for 40 minutes) for three months. Pain was assessed by means of a visual analog scale and of the Wisconsin brief pain questionnaire. Results show that TENS reduced shoulder pain in both patients. Treatment with TENS may hence be effective in reducing shoulder pain in patients with CKD.

KEY words: Amyloidosis; Electrical stimulation therapy; Renal insufficiency, chronic; Shoulder pain 


\section{INTRODUÇÃO}

A doença renal crônica (DRC) é definida como uma alteração da filtração glomerular (clearance de creatinina $<60$ $\mathrm{ml} / \mathrm{min} / 1,73 \mathrm{~m}^{2}$ ) por pelo menos três meses $^{1}$. Nas fases avançadas da DRC (estágio 5), os pacientes não sobrevivem sem a terapia de substituição renal, a hemodiálise. No entanto, apesar do enorme progresso que essa técnica alcançou nos últimos anos, os pacientes desenvolvem inúmeras complicações ${ }^{1}$, uma das quais, freqüente e precoce, é a alteração do metabolismo mineral que leva à osteodistrofia renal; a amiloidose é mais tardia e muito incapacitante ${ }^{2}$.

A amiloidose é uma doença decorrente do acúmulo de ß2-microglobulina que, posteriormente, se transforma em substância amilóide nos ossos e articulações. Esses depósitos favorecem inflamação e erosão da articulação, levando à dor e perda da movimentação osteoarticular $^{3}$. As principais manifestações clínicas da amiloidose são a síndrome do túnel do carpo, artropatia crônica e lesões císticas ósseas, sendo que o ombro e o punho são as articulações mais acometidas $^{3,4}$. Não há tratamento específico e o uso de analgésicos, anti-inflamatórios e corticosteróides são úteis no alívio dos sintomas, mas essas medicações apresentam efeitos colaterais severos ${ }^{4}$.

Tratamentos não-medicamentosos têm sido utilizados para redução das dores, especialmente nos ombros ${ }^{5} \mathrm{e}$, dentre estes, a TENS (estimulação elétrica nervosa transcutânea). Esse equipamento é usado no tratamento de dores agudas e crônicas há mais de 20 anos $^{6}$. Charbal et al. ${ }^{7}$ mostram que a TENS reduz a dor e conseqüentemente a necessidade de analgésicos e anti-inflamatórios nos pacientes com dor crônica. Até o presente, não foram encontrados relatos do uso da TENS para tratamento de dor no ombro em pacientes com DRC. O objetivo deste estudo é pois verificar o efeito da TENS no tratamento da dor no ombro em pacientes com DRC portadores de amiloidose.

\section{METODOLOGIA}

Foram estudadas duas pacientes (52 e 54 anos) com DRC em tratamento de hemodiálise por um longo período (13 e 24 anos, respectivamente), em seguimento no ambulatório de Osteodistrofia Renal do Hospital Universitário da Faculdade de Medicina da USP. Ambas foram orientadas e assinaram o termo de consentimento, após o estudo ser aprovado pelo Comitê de Ética do Hospital. As pacientes apresentavam relato de dor crônica incapacitante nos ombros e diminuição das atividades de vida diária secundária à amiloidose, confirmada pelo ultra-som de ombro. As principais alterações encontradas no ultra-som de ombro foram o depósito de amilóide no tendão supra-espinhal, erosão óssea na cabeça umeral, bursite subacromialsubdeltóidea e lesão parcial e ruptura de tendão supra-espinhal. As pacientes não eram gestantes nem usavam marcapasso, o que poderia ser contra-indicação para o uso da TENS. Ambas foram acompanhadas por um período de três meses, em que foram feitas quatro avaliações (inicial e após 1, 2 e 3 meses). Na primeira visita, a TENS foi aplicada no ombro das pacientes durante $40 \mathrm{mi}$ nutos e ao final da aplicação a paciente e seu cuidador eram orientados a respeito do uso do equipamento no domicílio. A paciente recebia o equipamento e retornava ao ambulatório três vezes a cada 30 dias. Nesse momento era reavaliada e, se necessário, reorientada. A cada retorno, a intensidade da dor era analisada utilizando-se uma escala visual analógica ${ }^{8}$ e o questionário de dor de Wisconsin ${ }^{9}$. Para a dor no ombro, as pacientes aplicaram apenas a TENS, tendo sido orientadas a não recorrer a outros tratamentos específicos exceto aos medicamentos prescritos pelos médicos.

A TENS foi aplicada durante 40 minutos com uma freqüência de $10 \mathrm{~Hz}$, duração do pulso de $150 \mu$ s e intensidade elevada, porém suportável pelo paciente. Os eletrodos foram colocados bilateralmente nos miótomos relacionados à articulação do ombro (deltóide e trapézio), que no caso eram a fonte de dor $^{10}$. As pacientes foram orientadas a utilizar o aparelho duas vezes ao dia (ao acordar e antes de dormir) e, a cada retorno mensal, o equipamento era novamente aplicado e a intensidade reajustada de acordo com a sensibilidade de cada uma delas.
A dor foi avaliada por uma escala visual analógica (EVA), que consiste em uma linha horizontal de dez centímetros com variações de zero (sem dor) a dez (dor insuportável) ${ }^{10}$, onde a paciente faz uma marca atribuindo uma "nota" para a dor no ombro; a distância (em centímetros) entre o ponto zero e a marca traçada pela paciente é então mensurada. Foi aplicado ainda o questionário de dor Wisconsin, que avalia a menor e a maior dor corpórea nas últimas 24 horas, além da dor naquele momento (também em EVA); o questionário inclui ainda duas questões, sobre medicamentos utilizados para o tratamento da dor e sobre o alívio da dor pelo uso de TENS, respondidas em uma escala de $0 \%$ a $100 \%$. Por fim, o questionário avalia o quanto a dor interfere nas atividades de vida diária9

Por se tratar de um relato de dois casos, os dados foram analisados de forma descritiva e os resultados são apresentados em média e desvio padrão.

\section{RESULTADOS}

Tal como avaliada pela EVA, a dor no ombro diminuiu em ambas as pacientes já no primeiro mês de tratamento (Gráfico 1). Na paciente 1 a dor foi se reduzindo ao longo do tratamento, desde insuportável (escore 10) até atingir o escore 1,2 após os 3 meses de tratamento. A paciente 2 relatou dor inicial de 3,8, reduzindo-se na segunda avaliação para 1,2 e mantendo-se nesse nível até o fim do tratamento.

A percepção de dor corpórea ao longo do tratamento, avaliada pelo questionário de Wisconsin, demonstrou sua redução em ambas as pacientes até o final do segundo mês de tratamento. Desse mês para o terceiro, entretanto, enquanto a paciente 1 relatou ausência total de dor corpórea (escore zero), a paciente 2 informou aumento de intensidade da dor (Gráfico 2).

As duas pacientes recorriam a medicação analgésica. A paciente 1 não modificou essa medicação (cloridrato de tramadol uma vez ao dia) ao longo do tratamento. Mas a paciente 2, que tomava dipirona sódica (uma vez ao dia) interrompeu seu uso ao final do segundo mês, ficando sem medicação. Na avalia- 
Dor no ombro (EVA)

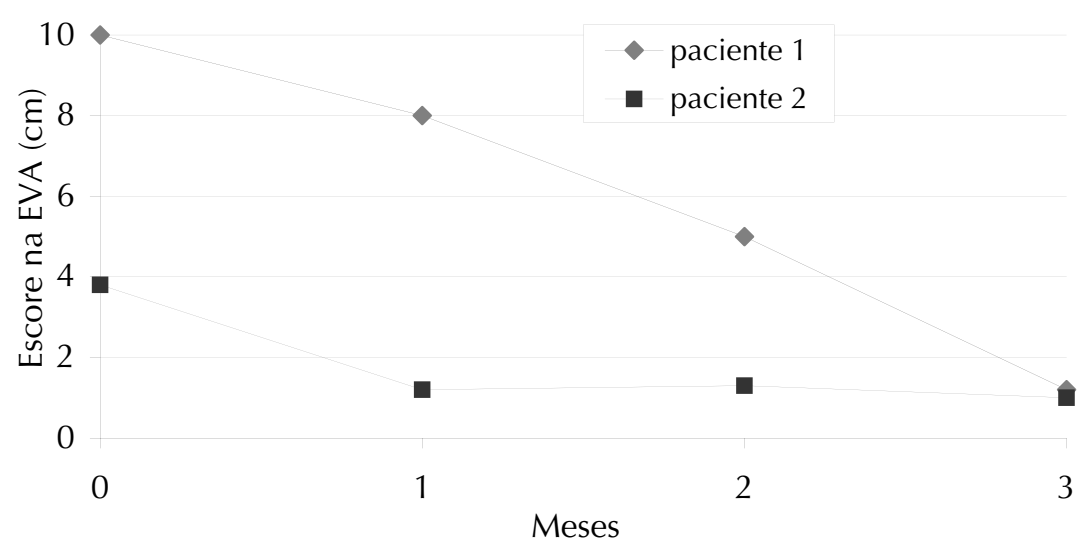

Gráfico 1 Evolução da dor no ombro nas duas pacientes, avaliada pela escala visual analógica (EVA)

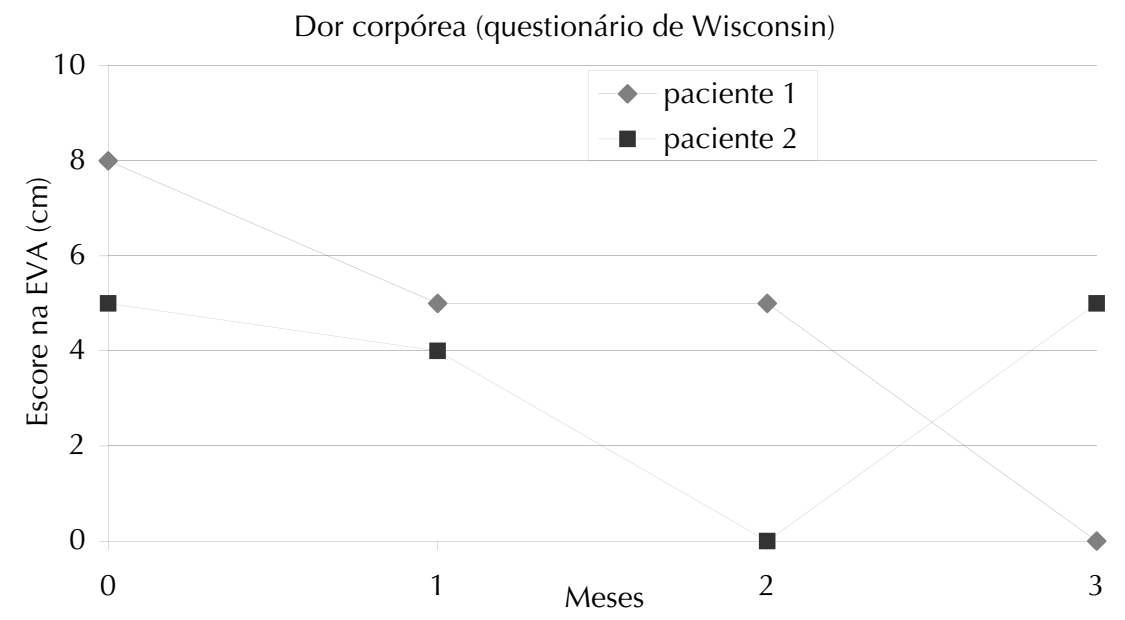

Gráfico 2 Evolução da dor corpórea nas duas pacientes, avaliada por escala visual analógica (EVA) do questionário de dor de Wisconsin

Alívio recente da dor

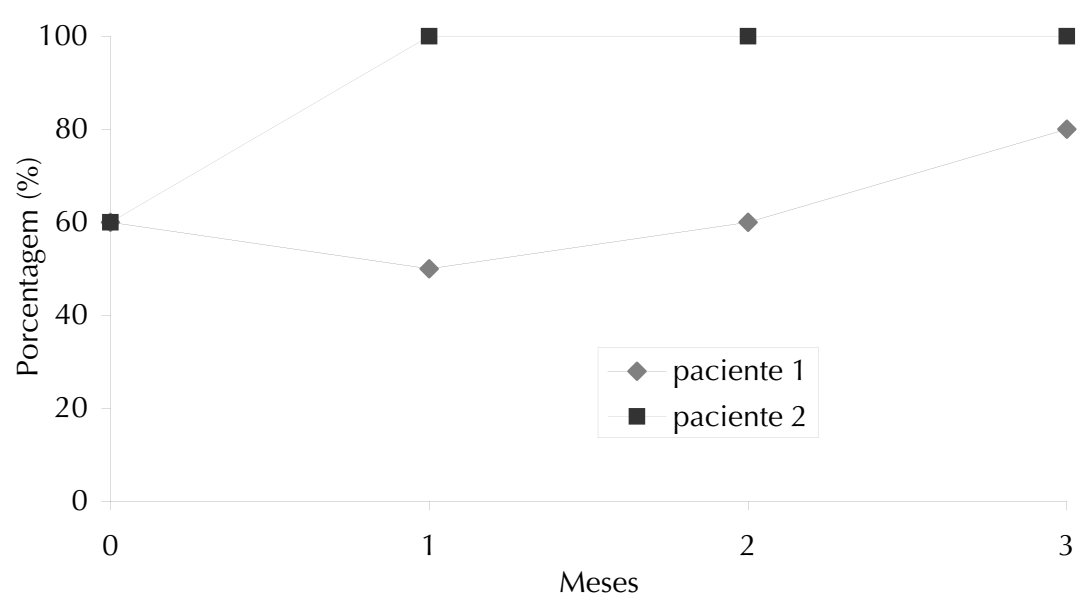

Gráfico 3 Alívio da dor (\%) relatado pelas duas pacientes no questionário de Wisconsin: por medicamentos nas $24 \mathrm{~h}$ anteriores àavaliação inicial e por medicamento e/ou TENS ao final do $1^{\circ}, 2^{\circ}$ e $3^{\circ}$ meses de tratamento ção inicial pelo questionário de Wisconsin, ambas indicaram que esse tratamento melhorava em 60\% sua dor (Gráfico 3). Ao final do primeiro mês, a paciente 1 relatou que o uso da TENS associada a analgésicos aliviou a dor em 100\% (ausência total de dor), tendo esse quadro permanecido até o final do tratamento (Gráfico 3). A paciente 2 não relatou melhora da dor até o segundo mês, apenas ao final do terceiro mês (Gráfico 3). Essa paciente relatou ainda que a meIhora da dor influenciou suas atividades diárias após os três meses de tratamento, com melhora também do humor e da qualidade do sono.

\section{DISCUSSÃOE CONCLUSÃO}

Os resultados indicam que o tratamento com TENS melhora a dor no ombro de pacientes com doença renal crônica (DRC) decorrente da amiloidose. Esse resultado é relevante porque se desconhecem, até o presente, estudos sobre o uso dessa modalidade terapêutica em pacientes com DRC, sugerindo que o fisioterapeuta pode ter um papel importante no tratamento de doentes com DRC e amiloidose.

A amiloidose relacionada à diálise é uma síndrome que pode levar à incapacidade, tal a severidade da dor ${ }^{4}$. A dor no ombro decorrente do depósito de amilóide nos tecidos ocorre pela infiltração de células inflamatórias que favorece o desenvolvimento de bursite, tenossinovite e também erosão óssea ${ }^{3}$. No presente estudo, a presença de amiloidose foi avaliada pelo exame ultrassonográfico. Existem relatos de diversos tratamentos para a dor no ombro, como antiinflamatórios não-esteroidais, acupuntura e cirurgia. Uma revisão sistemática da literatura avaliou a efetividade de diversas técnicas de fisioterapia no tratamento da dor no ombro, dentre as quais exercícios terapêuticos, laser, ultra-som e TENS, concluindo que essas modalidades têm uma efetividade pouco estabelecida para o tratamento de dor no ombro e que são necessários mais estudos para melhor compreensão dessas técnicas terapêuticas ${ }^{11}$. Apesar de não haver consenso sobre a efetividade dessas técnicas para o tratamento da dor 
no ombro ${ }^{5}$, a TENS foi a opção terapêutica no presente estudo porque é um tratamento não-invasivo, seguro e de fácil aplicação ${ }^{12}$. Além disso, como o uso da TENS não apresenta efeitos colaterais as pacientes podiam utilizá-lo na própria residência: as pacientes estavam em hemodiálise e não dispunham de tempo para comparecer diariamente ao hospital.

As pacientes estudadas relatavam dor de moderada a muito intensa no ombro antes do tratamento (3,8 e $10 \mathrm{~cm}$ na EVA) e, após o tratamento, houve uma meIhora importante, tendo ambas indicado escores próximos a $1 \mathrm{~cm}$. Supõe-se que o mecanismo de redução da dor nas pacientes, embora não tenha sido estudado, se deva à liberação de opióides endógenos, efeito sugerido pelo uso da TENS de baixa freqüência ${ }^{12}$. Como as pacientes apresentavam dor crônica no ombro, que as impedia de realizar atividades diárias como lavar roupa e pentear o cabelo, optou-se pelo uso da TENS de baixa freqüência porque essa modalidade parece ter um efeito analgésico mais duradouro, observado em pacien- tes com dor lombar crônica ${ }^{13}$ e em modelos animais ${ }^{14}$. Entretanto, não se pode assegurar que a TENS de alta freqüência não desencadeie os mesmos efeitos, visto que alguns estudos sugerem que tanto a TENS de alta quanto de baixa freqüência podem reduzir dores crônicas ${ }^{15-17}$. Isso poderá ser avaliado em estudos futuros. Existem evidências de que a TENS produz alívio acentuado da dor em pacientes com dores musculoesqueléticas (lombalgia crônica e aguda, artrite reumatóide e osteoartrite $)^{18}$. Sluka e Walsh ${ }^{12}$ sugerem que a TENS permite aos pacientes aumentar seu nível de atividade, diminuir o tempo de hospitalização e melhorar a função articular. Acredita-se que a diminuição da dor e o aumento da função articular favorecem o uso de outras terapias, além de facilitar outras atividades, resultando na melhora da qualidade de vida. É relevante o dado de que as duas pacientes relataram (nas respostas ao questionário de Wisconsin) que a redução da dor permitiu realizar atividades da vida diária. E relataram também melhora do humor e da qualidade do sono.
Segundo as respostas ao questionário de Wisconsin, a paciente 2 teve um aumento da dor corpórea no terceiro mês de tratamento. Entretanto, essa piora não deve ter decorrido do uso da TENS, pois a paciente havia relatado piora de uma antiga lesão no joelho. Essa informação foi mantida porque se entende que não interfere na relevância dos resultados. Não existe uma maneira única de avaliar dor específica para o ombro. Foi feita a opção pelas escalas EVA e Wisconsin porque a primeira é a mais utilizada para avaliar a eficácia de tratamentos para dor ${ }^{8}$ e o segundo avalia outros fatores, como a interferência da dor nas atividades de vida diária e uso de medicamentos analgésicos ${ }^{9}$.

A melhora da dor observada pelo tratamento com TENS não pode ser explicada por alteração da medicação analgésica, porque uma paciente relatou interrupção do uso de analgésico enquanto a outra o manteve. É interessante notar que a redução do uso de analgésicos com o tratamento pela TENS também foi mostrada em estudo de pacientes com dor crônica ${ }^{8}$.

\section{REFERÊNCIAS}

1 Levey AS, Eckardt K, Tsukamoto Y, Levin A, Coresh J, Rossert J, et al. Definition and classification of chronic kidney disease: a position statement from kidney disease; improving global outcomes (KDIGO). Kidney Int. 2005;67:2089-100.

2 Freemont AJ. The pathology of dialysis. Semin Dial. 2002;15:227-31.

3 Danish F, Ho LT. Dialysis-related amyloidosis: history and clinical manifestation. Semin Dial. 2001;14:80-5.

4 Nangaku N, Miyata T, Kurokawa K. Pathogenesis and management of dialysis-related amyloid bone disease. Am J Med Sci. 1999;317:410-5.

5 Philadelphia Panel Members. Philadelphia panel evidence-based clinical practice guidelines on selected rehabilitation interventions for shoulder pain. Phys Ther. 2001;81:1719-30.

6 Lampl C, Kreczi T, Klinger D. Transcutaneous electrical nerve stimulation in the treatment of chronic pain: predictive factors and evolution of the method. Clin J Pain. 1998; 14:134-42.
7 Charbal C, Fishbain DA, Weaver MMA, Heine LW. Long-term transcutaneous electrical nerve stimulation (TENS) use: impact on medication utilization and physical therapy costs. Clin J Pain. 1998;14:66-73.

8 Jensen MP, Karoly P, Braver S. The measurement of clinical pain intensity: a comparison of six methods. Pain. 1986;27:117-26.

9 Daut RL, Cleeland CS, Flanery RC. Development of the Wisconsin Brief Pain Questionnaire to assess pain in cancer and other diseases. Pain. 1983;12:197-210.

10 Robinson AJ, Snyder-Mackler L. Eletrofisiologia clínica. 2a ed. Porto Alegre: Artmed; 2002.

11 Green S, Buchbinder R, Hetrick S. Physiotherapy interventions for shoulder pain. Cochrane Library, 2003 [citado mar 2008]. Disponível em: http:ovidsp.tx.ovid.com/spb/ovidweb.cgi..

12 Sluka K, Walsh D. Transcutaneos electrical nerve stimulation: basic science mechanisms and clinical effectiveness. J Pain. 2003;4:109-21. 
13 Warke K, Al-Smadi J, Baxter D, Walsh DM, Lowe-Strong A. Efficacy of transcutaneous electrical nerve stimulation (TENS) for chronic low-back pain in multiple sclerosis population. Clin J Pain. 2006;22:812-9.

14 Sabino GS, Santos CMF, Francischi JN, Resende MA. Release of endogenous opioids following transcutaneous electric nerve stimulation in an experimental model of acute inflammatory pain. J Pain. 2008;9:157-63.

15 Grimmer K. A controlled double-blind study comparing the effects of strong burst mode TENS and high rate TENS on painful osteoarthritis knees. Aust J Physiother. 1992;48:49-56.
16 Jensen H, Zester R, Christensen T. Transcutaneous electrical nerve stimulation (TNS) for painful osteoarthrosis of the knee. Int J Rehabil Res. $1991 ; 14: 356-8$.

17 Tulgar M, McGlone F, Bowsher D, Miles JB. Comparative effectiveness of different stimulation modes in relieving pain, part I: a pilot study. Pain. 1991;47:151-5.

18 Robinson AJ. Transcutaneous electrical nerve stimulation for the control of pain in musculoskeletal disorders. J Orthop Sports Phys Ther. 1996;24:208-26. 